\title{
MR-Guided Interventions for Prostate Cancer
}

\author{
Ergin Atalar, $\mathrm{PhD}^{\mathrm{a}, \mathrm{b}, *}$, Cynthia Ménard, $\mathrm{MD}^{\mathrm{c}}$ \\ ${ }^{a}$ Departments of Radiology, BME, and ECE, The Johns Hopkins University, 720 Rutland Avenue, \\ Traylor 330, Baltimore, MD 21205, USA \\ ${ }^{\mathrm{b}}$ Electrical and Electronics Engineering Department, Bilkent University, Ankara 06800, Turkey \\ ${ }^{\mathrm{c}}$ Princess Margaret Hospital, University Health Network, University of Toronto, 5th Floor, \\ 610 University Avenue, Toronto, Ontario, M5G 2M9, Canada
}

With an estimated annual incidence of 230,000 cases in 2004, prostate cancer is the most common noncutaneous cancer in men in the United States [1]. Despite an impressive scope of research efforts, difficult challenges persist in various aspects of prostate cancer care, including diagnosis, prognostication, and treatment.

First and foremost is a pressing need to appropriately tailor therapeutic interventions to the spatial extent and biologic aggressiveness of disease for individual patients. Individualized therapy for localized disease could significantly reduce the treatment-related morbidity incurred by this population of patients but requires the development of better measures to delineate and characterize disease. At present, these measures are limited to nonspecific prostate-specific antigen (PSA) serum levels [2], histopathologic estimates of tumor burden, and Gleason grading, which is subject to random biopsy sampling error [3-5] and insensitive digital rectal examinations that are poorly reproducible among observers [6].

The authors believe that in the near future, imaging will bridge the gap between characterization of disease and individualized therapy. An ability to visualize the complete extent and biologic profile of prostate cancer with regard to

This work was supported in part by the National Science Foundation grant NSF ERC9731478, the US Army grant PC10029, and the National Institutes of Health grants R01 HL 57483 and R01 HL 61672.

* Corresponding author. Departments of Radiology, BME, and ECE, Johns Hopkins University, 720 Rutland Avenue, Traylor 330, Baltimore, MD 21205.

E-mail address: eatalar@jhu.edu (E. Atalar). prostatic anatomy may counter biopsy sampling error, enable appropriate patient selection for local therapy, guide local therapy to the disease rather than to the entire prostate gland, and provide a noninvasive means of monitoring progression or response to therapy.

Here, the authors review the studies that are currently investigating the potential role of MR imaging in guiding needle-based prostate interventions. This review omits studies that use MR images for guidance of external beam radiotherapy [7] and, instead, focuses primarily on interventional procedures that are conducted in the MR imaging scanner room. The article starts with a brief overview of the role of diagnostic MR imaging in prostate cancer.

\section{MR imaging for prostate cancer}

It is unfortunate that there is no single imaging method that embodies all of the optimal characteristics for the integration of diagnostic and interventional procedures for prostate cancer. CT permits accurate spatial visualization of interventional devices (Fig. 1A) but does not provide real-time feedback or adequate soft tissue delineation. Transrectal ultrasound (TRUS) is the current "gold standard" for guiding prostate interventions due to its ease of use and real-time image feedback. Soft tissue delineation is better with TRUS than with CT, but most tumors are not visible under ultrasound, and biologic profiling is currently limited [8]. In addition, accurate visualization of interventional needles remains challenging (see Fig. 1B). The interventional 

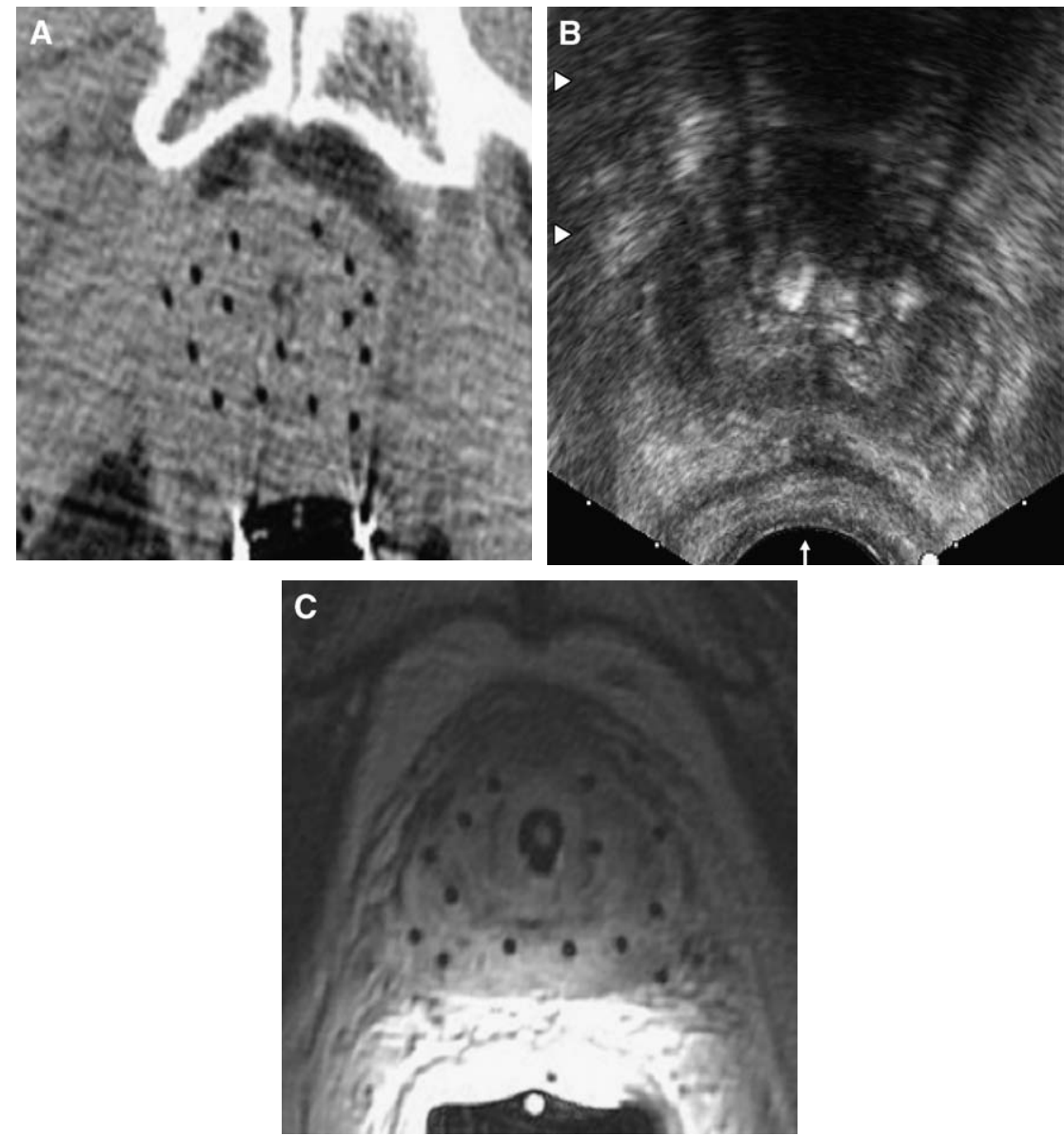

Fig. 1. Interventional images during high dose rate brachytherapy show the superiority of MR imaging in depicting the prostate anatomy and the interventional catheters. (A) CT scan image. (B) Transrectal ultrasound image. (C) MR image.

needles and the prostate anatomy are clearly visible in MR images (see Fig. 1C). From this perspective, MR imaging is well suited for guidance of interventional procedures. The principal limitations to its routine use include a lack of realtime feedback and a complex and technically challenging environment.

A number of anatomic structures can be clearly delineated on MR imaging, including the distal prostatic urethra (up to the point of insertion in the central gland), the central zone, the peripheral zone, the prostatic capsule, the levator ani, and the rectal mucosa [9]. On contrastenhanced MR images, the neurovascular bundles can also be identified (Fig. 2) [10]. Diagnostic MR imaging, however, has the potential to provide more than high image resolution of the prostate anatomy. Endorectal coil MR imaging of the prostate gland has demonstrated value for staging and prognostication in patients with localized disease [11-16]. When T2-weighted anatomic images, which are sensitive but not specific for malignancy, are combined with biologic imaging techniques such as MR spectroscopic imaging [17-20] and dynamic contrast-enhanced (DCE) MR imaging [8], MR imaging may be able to accurately identify predominant subsites of tumor burden.

DCE MR imaging is a promising tool for visualizing the vascular physiology of solid tumors. With the advent of modern multislice imaging techniques and data analysis tools, imaging the entire prostate gland with high spatial and temporal resolution using DCE MR imaging is 


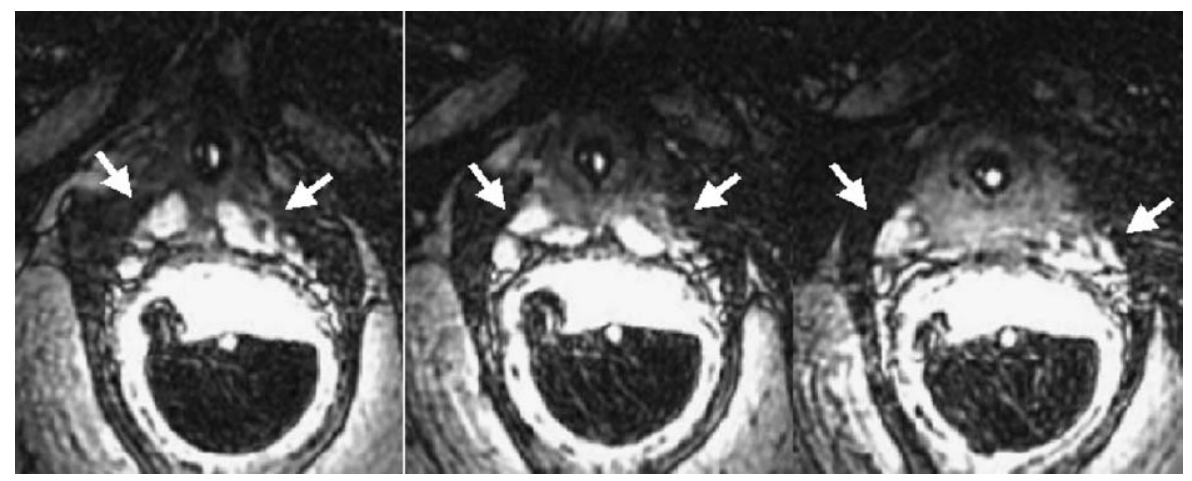

Fig. 2. Neurovascular bundle imaging. The neurovascular bundle (arrows) along the prostate gland can be visualized post contrast three-dimensional TrueFISP images. Each panel represents contiguous axial slices at the prostate apex. (From Citrin D, Ning H, Guion P, et al. Inverse treatment planning based on MRI for high dose rate prostate brachytherapy. Int J Radiat Oncol Biol Phys 2005;61(4):1272; with permission.)

feasible. In a recent study [8], the sensitivity and specifity of DCE MR imaging for localizing malignancy in the peripheral zone were estimated to be $87 \%$ and $74 \%$, respectively, based on a subjective coregistration to TRUS-guided biopsies. This level of accuracy is not maintained in the central gland (sensitivity $96 \%$, specificity $46 \%$ ) because coexisting benign prostatic hyperplasia is also characterized by high vascularity.

Kurhanewicz and colleagues [17] proposed a three-dimensional MR spectroscopic imaging technique for the detection of prostate cancer. This promising method compares the ratios of choline and creatine to citrate peak levels as a marker of malignancy in the peripheral zone. One of the main limitations of this MR spectroscopic imaging technique is the relatively long data acquisition time and the low signal-to-noise ratio inherent to spectroscopy. The use of large voxels in the imaging protocol partially solves this problem but introducs partial volume effects whereby small lesions may become invisible.

In addition to the techniques of T2-weighted MR imaging, DCE MR imaging, and MR spectroscopic imaging, there are a number of complementary techniques under investigation to improve the diagnostic accuracy of MR imaging. For example, tissue hypoxia, a known biomarker associated with prostate cancer, can be interrogated using blood oxygen level-dependent imaging techniques [21,22]. Diffusion maps of the prostate gland can also be generated with MR imaging, thus providing noninvasive information related to interstitial fluid pressure changes in normal and malignant prostate tissue [23,24].

\section{Needle core biopsy}

Currently, prostate biopsy is conducted under TRUS guidance. Although a positive biopsy result is a clear indication of cancer, a negative biopsy result is often indefinite and problematic because it is known that the sextant biopsy procedure has a relatively low sensitivity and high sampling error [25]. To address this problem, an 8 - to 10-biopsy regimen, depending on prostate size, has been proposed [26], with sensitivity increasing up to $80 \%$. Repeat sextant biopsy is another approach, which further increases the sensitivity of this approach [27]. Image-guided biopsy may be the best approach to this problem, but ultrasound can be blind to $40 \%$ of lesions, which are isoechoic [28].

MR-guided biopsy may have an immediate impact by improving the sensitivity of needle core biopsies to detect prostate cancer, specifically for those $20 \%$ of patients who have false-negative biopsy results from sampling error when performed under TRUS guidance [29]. By combining tissue biopsy with MR imaging (ie, to directly biopsy tissue regions with a suspicious MR imaging appearance), the high sensitivity of MR imaging [30] may be obtained while gaining the specificity of tissue biopsy.

In addition, MR imaging guidance of needle biopsies is a critical step in the histopathologic validation of emerging MR imaging techniques for prostate cancer delineation and characterization. These new imaging techniques must be validated against gold standard measures to establish their accuracy, and in this case, the gold 
standard is prostate biopsy and histopathology. Notable intraprostatic [31] and intratumoral [32] biologic heterogeneity mandates millimeter colocalization accuracy between tissue samples and their corresponding image pixels. When prostate MR imaging and tissue acquisition procedures are performed in different settings and at different times, however, spatial coregistration is fraught with error.

Stereotactic needle placement under MR imaging guidance enables two critical steps in the coregistration of tissue and MR imaging data. First, it directly guides biopsies to sites of suspected tumor on MR imaging, and second, it permits volumetric verification and documentation of the actual biopsy location with regard to MR imaging data.
Investigators at Harvard University were the first to report MR-guided prostate biopsies, which were performed in patients with suspicion of prostate cancer who were not candidates for the standard TRUS-guided technique because of a previous proctocolectomy [33,34]. Using an open-configuration 0.5-T MR imaging scanner and a pelvic coil, transperineal needle core biopsies were performed with patients in the dorsal lithotomy position. Sites deemed suspicious for cancer on previously acquired diagnostic MR imaging were subjectively correlated to corresponding sites on the interventional MR imaging images and specifically targeted through a stereotactically registered perineal template (Fig. 3). A nonconventional transgluteal approach has also been reported using an open low-field MR
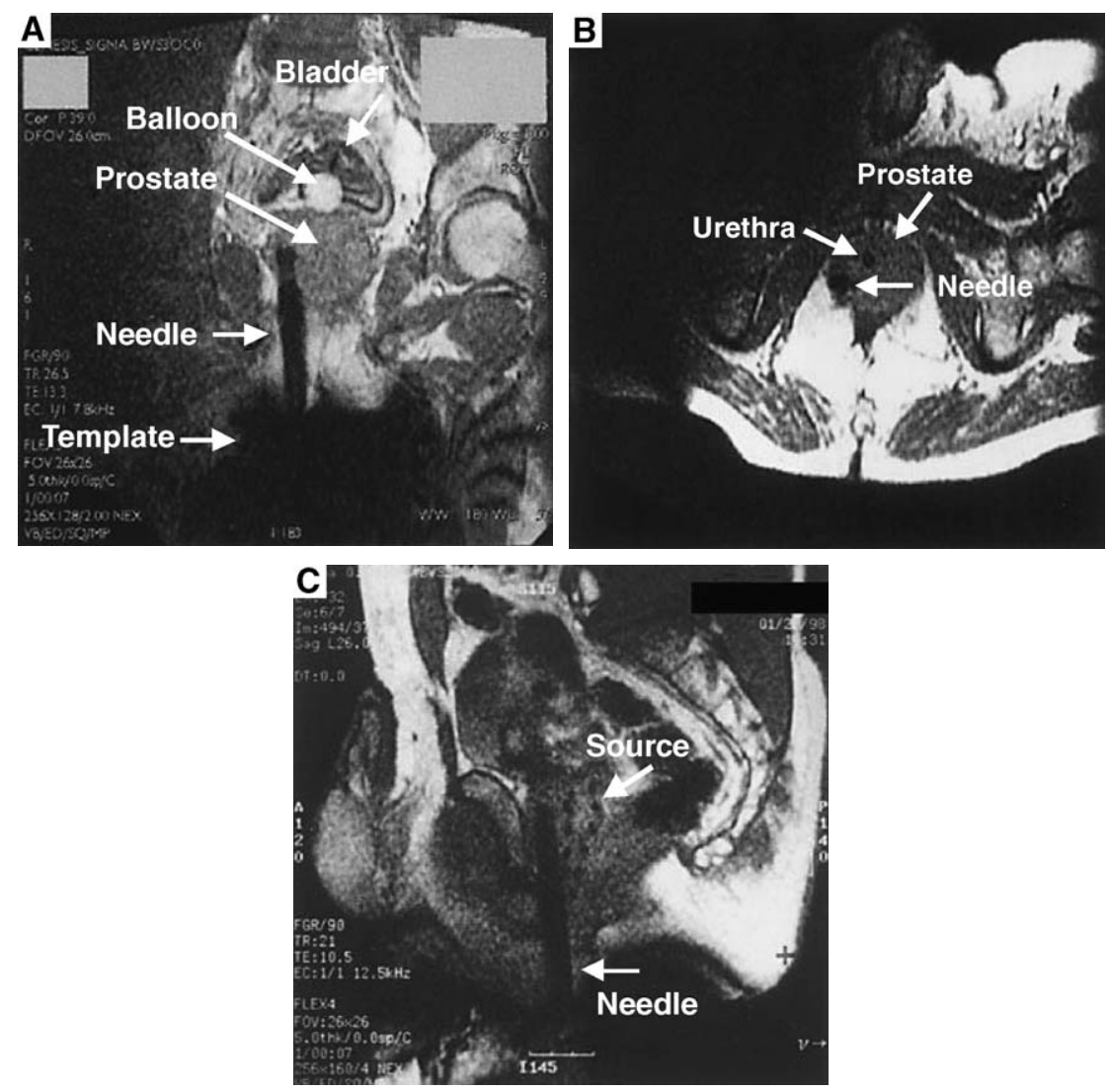

Fig. 3. Images obtained during MR-guided diagnosis and treatment of prostate cancer in a 62-year-old man. $(A)$ Coronal view of the prostate gland and bladder. The tip of the biopsy needle has been placed through the perineum and into the lesion, located in the right midportion of the gland. The template was used for accurate placement of the needle. $(B)$ Axial view of the prostate gland with the tip of the biopsy needle in the right midportion of the gland. $(C)$ Real-time intraoperative catheter placement in the sagittal plane during MR-guided prostate brachytherapy. The black spots are previously deposited radioactive sources posterior to the needle. (From D'Amico AV, Cormack RA, Tempany CM. MRI-guided diagnosis and treatment of prostate cancer. N Engl J Med 2001;344(10):776; with permission.) 
imaging scanner in patients with uncertain or suspicious prostate lesions on diagnostic MR imaging [35]. Diagnostic images were similarly subjectively correlated to interventional MR images to define biopsy target sites. Using T1weighted sequences, 25 biopsy procedures were performed successfully with MR guidance in all cases without any side effects or complications. Alternatively, diagnostic MR images have been rigidly coregistered to interventional ultrasound images for guidance [36].

To circumvent the need for and the error associated with deformable or rigid registration of previously acquired diagnostic MR images, transperineal biopsies have been performed under direct MR imaging guidance in a cylindric 1.5-T scanner [37]. To address the challenge of accessing the perineum under the geometric constraint of a $60-\mathrm{cm}$ diameter bore, patients were positioned in the left lateral decubitus position (Fig. 4). Biopsies were again performed through a stereotactically registered perineal template that in this case was affixed perpendicularly to a rigid endorectal coil, thereby increasing signal-to-noise ratio and image quality. The mean biopsy-needle targeting accuracy of the stereotactic system was $2.1 \mathrm{~mm}$.

Finally, two competing devices for transrectal prostate biopsy in a cylindric 1.5-T scanner have recently been developed [38,39] and clinically tested in patients with prostate cancer $[39,40]$. The main advantage of the transrectal approach is a shorter needle path length, which translates to less tissue trauma and patient discomfort. For access, patients are positioned prone on the MR imaging table.

The first MR-guided transrectal biopsy system was developed at Charité, Humboldt-Universität zu Berlin in cooperation with MRI Devices/Daum (Schwerin, Germany). This device is made of polyoxymethylene and consists of a base plate, an adjustable arm, and a needle guide filled with contrast material gel that can be visualized on MR imaging. After the patient is positioned, the needle guide is inserted into the rectum and connected to the arm of the biopsy device (Fig. 5). The arm enables the needle guide to be rotated, translated
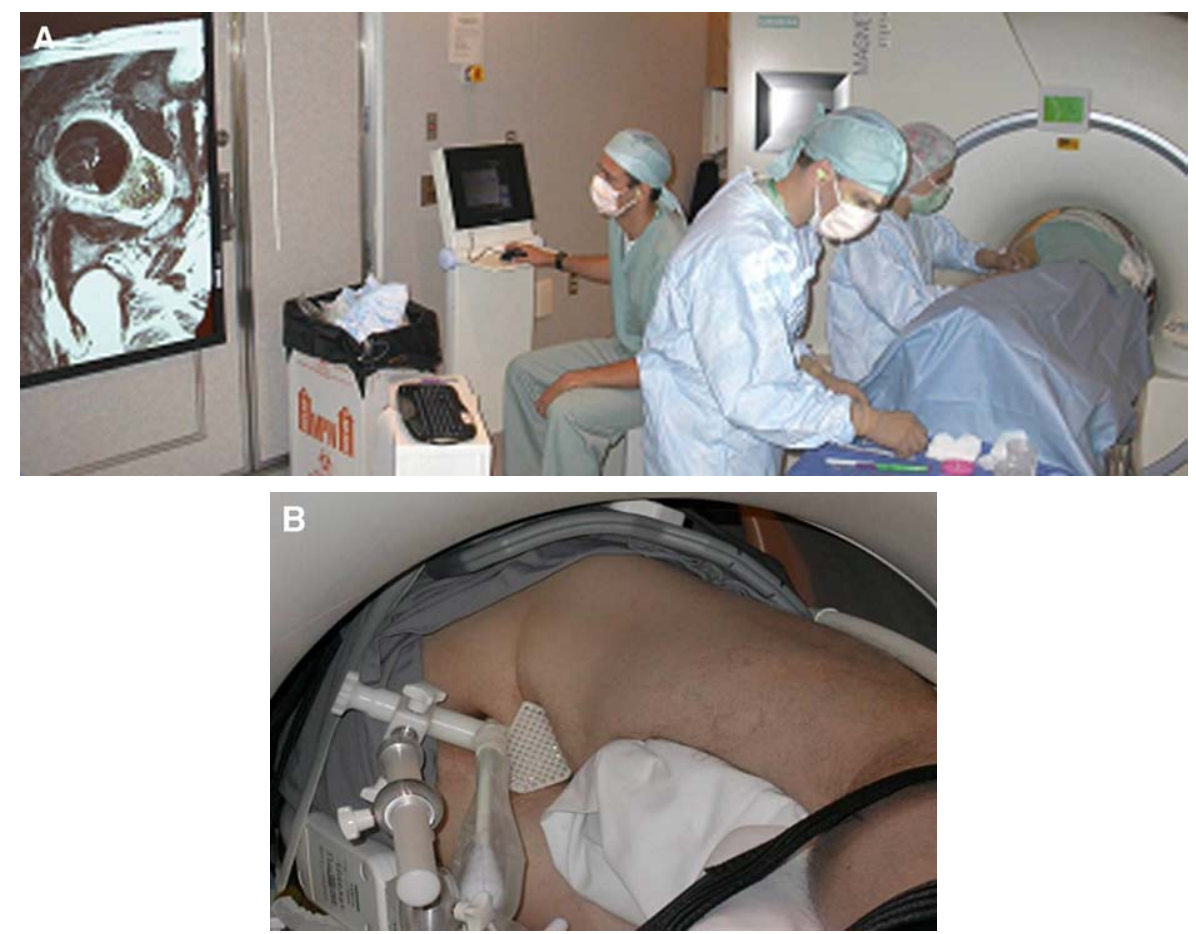

Fig. 4. Setup for transperineal biopsy in a conventional 1.5-T MR imaging scanner. $(A)$ Prostate images are displayed within the scanner room using stereotactic targeting software. $(B)$ The patient is positioned in the left lateral decubitus position. An endorectal coil is affixed perpendicular to the perineal template. (From Menard C, Susil RC, Choyke P, et al. MRI-guided HDR prostate brachytherapy in standard 1.5T scanner. Int J Radiat Oncol Biol Phys 2004;59(5):1417; with permission.) 


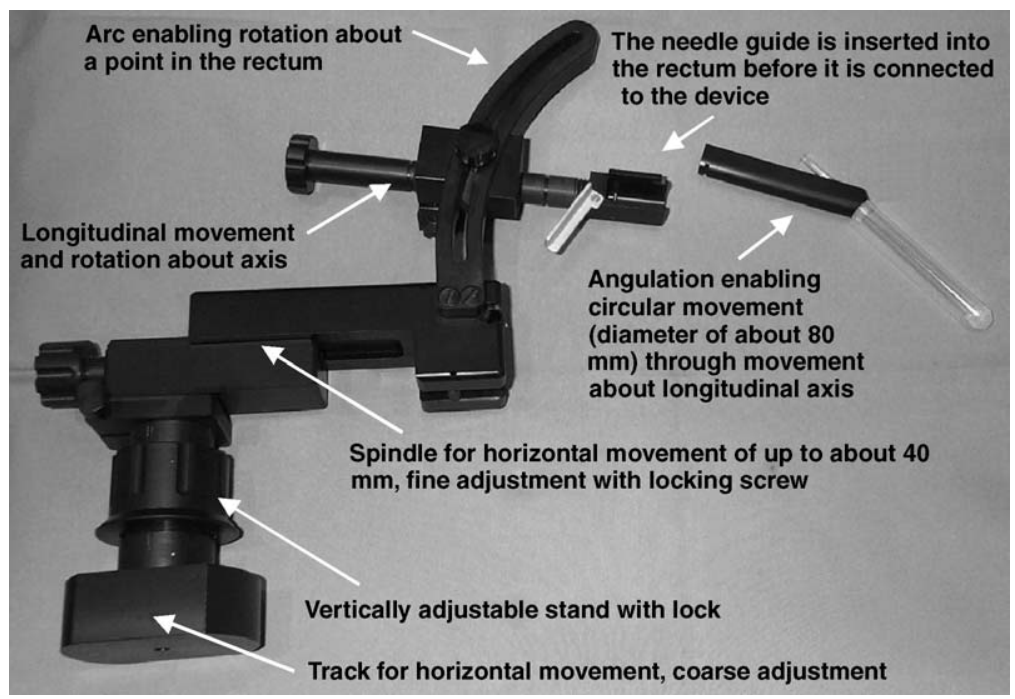

Fig. 5. The endorectal biopsy system developed at Charité, Humboldt-Universität zu Berlin in cooperation with MRI Devices/Daum. Passive markers are imaged to register the device with respect to the prostate. (From Beyersdorff D, Winkel A, Hamm B, et al. MR imaging-guided prostate biopsy with a closed MR unit at 1.5 T: initial results. Radiology 2005;234(2):577; with permission.)

forward and backward, and adjusted in height. In addition, the insertion angle can be changed by rotating the needle guide about a point inside the rectum.

In the initial study, biopsies were obtained from suspicious areas of the prostate (Fig. 6) in 12 patients by means of an MR imaging-compatible automatic $(n=5)$ or semiautomatic $(n=7)$ 16-gauge core needle biopsy device (Double-Shoot Biopsy Gun or Semi-Automatic Biopsy Gun; MRI Devices/Daum). The authors reported that of the 16 biopsy specimens from areas that were highly suspicious for prostate cancer at prebiopsy MR imaging, 8 were positive and 8 were negative. Of the 24 biopsy specimens from moderately suspicious areas, 4 showed prostate cancer and 20 showed no prostate cancer. Of the 57 specimens from nonsuspicious areas, 2 showed prostate cancer and 55 did not.

It is important to note that the investigators did not use DCE MR imaging or MR spectroscopic imaging to identify the suspected tumor
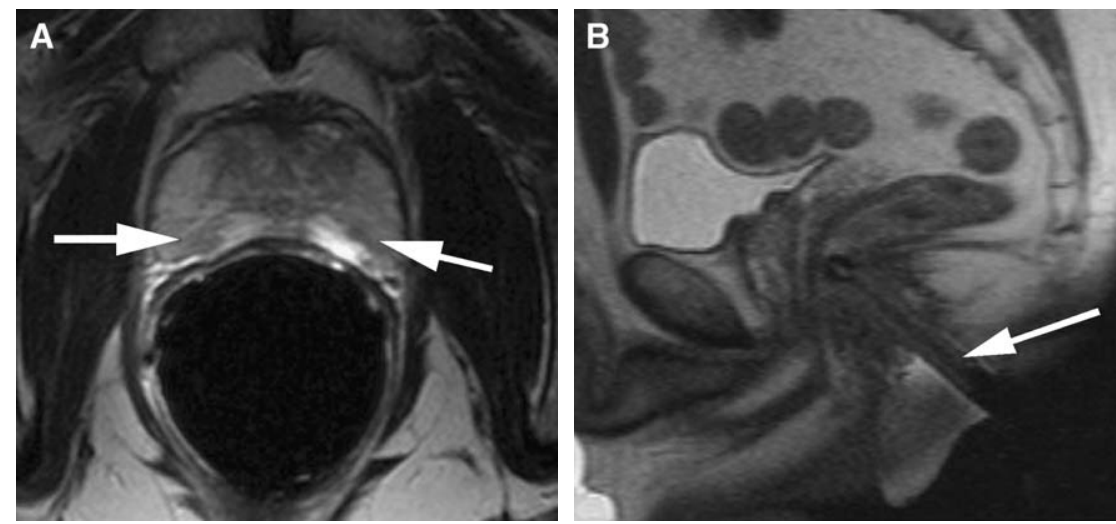

Fig. 6. (A) Axial T2-weighted diagnostic MR image of the prostate showing suspicious lesions (arrows). (B) Sagittal interventional image of the endorectal biopsy procedure. The arrow shows the location of the biopsy device. (From Beyersdorff D, Winkel A, Hamm B, et al. MR imaging-guided prostate biopsy with a closed MR unit at $1.5 \mathrm{~T}$ : initial results. Radiology 2005;234(2):579; with permission.) 
locations; however, they demonstrated a very strong correlation between MR imaging findings and biopsy results. These results are very encouraging for the future widespread use of MR-guided biopsy procedures.

The other transrectal biopsy device, the "access to prostate tissue under MR imaging guidance" (APT MR imaging) system (Fig. 7) [38,40], consists of a $23-\mathrm{mm}$ diameter hollow endorectal sheath, placed at the beginning of the procedure, that remains immobile throughout the intervention. The sheath includes an integrated $20-\mathrm{mm}$ diameter single-turn imaging coil surrounding an anterior window that allows for needle access to the prostate. An 18-mm diameter cylindric needle guide fits inside the stationary rectal sheath and contains three MR tracking microcoils (allowing for device registration). Needle channels at $20^{\circ}$ and $30^{\circ}$ of angulation permit transrectal needle access to the prostate gland. The cylindric needle guide is mounted on a positioning stage containing the mechanism that converts the rotation of two flexible control rods-each extending to the edge of the scanner bore-into the rotation and translation of the needle guide. Finally, the positioning stage is attached to an immobilization arm mounted on a linear rail.

Four MR-guided prostate biopsy procedures have been reported to date with the APT MR imaging system for the histomolecular validation of DCE MR imaging [41]. Biopsy locations were selected throughout the peripheral zone of the prostate using T2-weighted fast spin-echo images and DCE MR images. Subsequently, after inserting the biopsy needle but before collecting the tissue core biopsy, T1-weighted fast spin-echo images were acquired to confirm biopsy needle placement accuracy. Fifteen tissue biopsies were collected; the mean biopsy needle placement accuracy was $1.8 \mathrm{~mm}$ (maximum error, $4.0 \mathrm{~mm}$ ) [41]. All biopsy cores were suitable for histologic evaluation and for genomic and proteomic microarray profiling. These data demonstrate the feasibility and value of stereotactic biopsies under MR imaging guidance and verification to provide a platform for rigorous histopathologic and biologic validation of MR imaging techniques (Fig. 8).

The APT MR imaging system has also been adapted to a 3-T MR imaging scanner and tested in six patients to date [41]. Because higher field strength translates to higher MR signal, the authors expect an improvement in the resolution of diagnostic images.

In summary, five different techniques of MRguided prostate biopsy have been reported in the clinical literature. Given the need and rationale for needle guidance to be based on diagnosticquality MR images, the authors favor a stereotactic approach without real-time image guidance within a diagnostic scanner. One of the limitations to a broader application of the latter cylindric scanner techniques relates to the instability and discomfort associated with the left lateral decubitus and prone positions. Although spatially accurate and robust, stereotactic guidance systems mandate an immobile prostate gland. A number of studies have shown that prostate motion is greatly reduced when patients are positioned supine, stemming from greater patient comfort and reduced respiratory motion [42-44]. Supine immobilization and perineal access in the cylindric

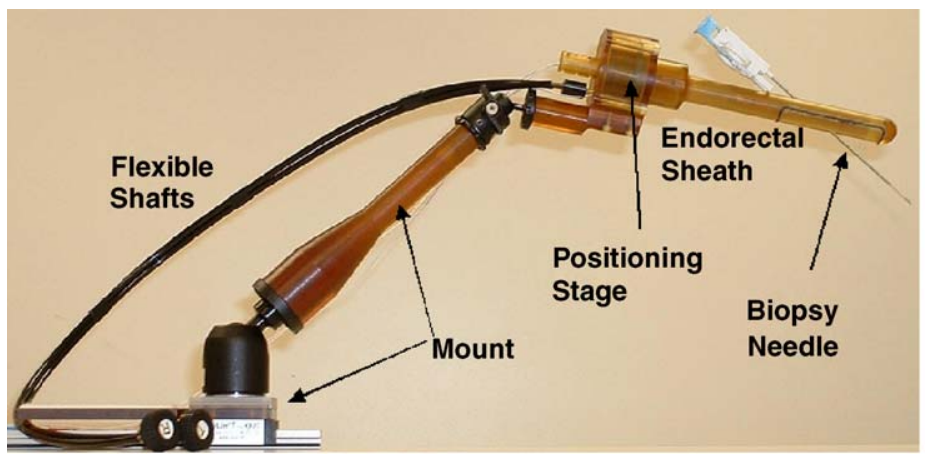

Fig. 7. The APT MR imaging system developed at the Johns Hopkins University. A stationary sheet minimizes the motion of the prostate during rotation and translation of the needle guide. The position of the needle guide is determined by active tracking coils. 


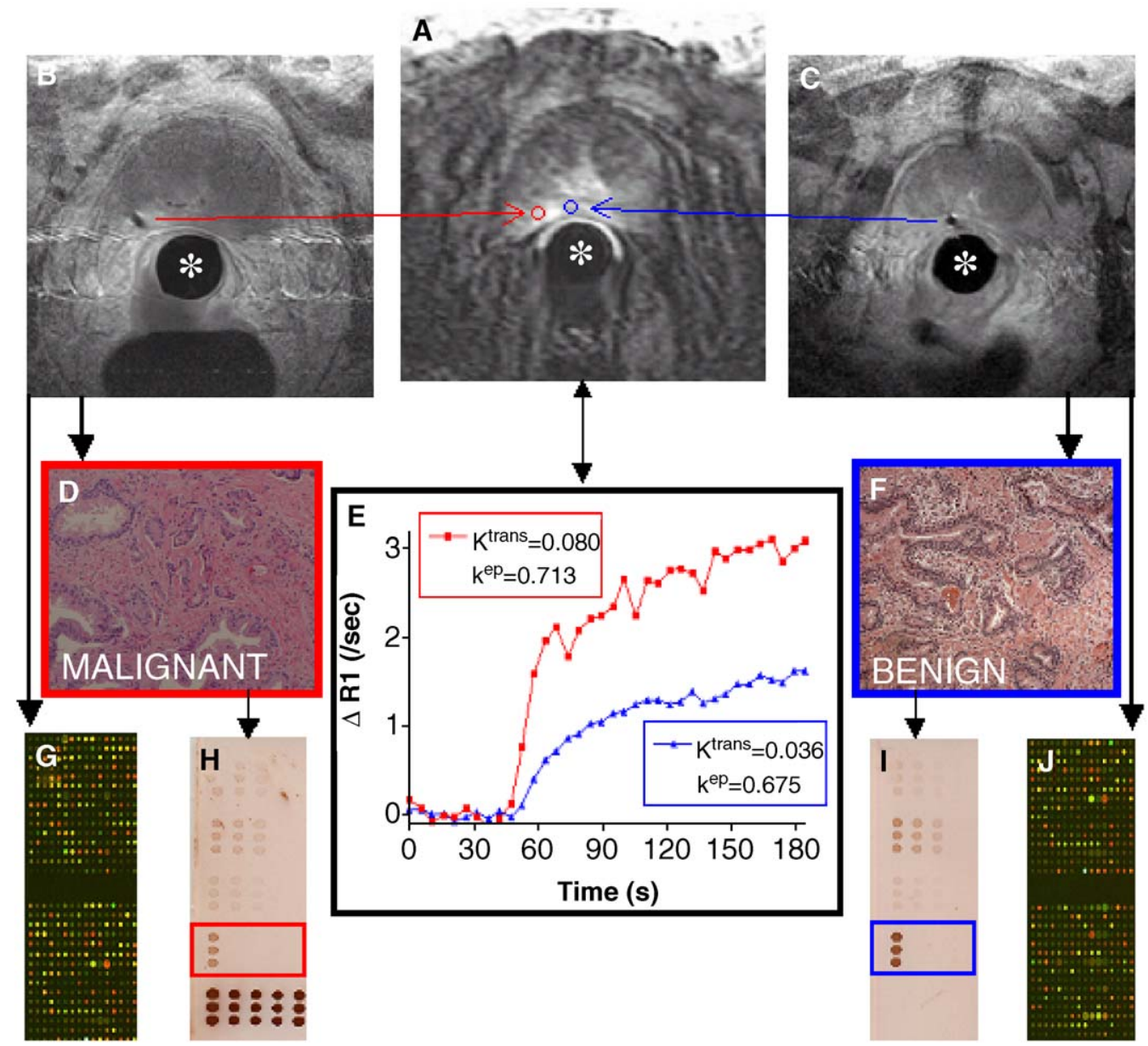

Fig. 8. Case example demonstrating the feasibility and integration of prostate interventional MR imaging for the correlation of molecular biology and DCE MR imaging. The stationary interventional endorectal coil (*) is used for diagnostic and interventional MR imaging. $(A)$ DCE MR imaging at 120 seconds shows a small area of increased signal intensity in the left peripheral zone of the prostate. Regions of interest (ROIs; red and blue) corresponding to the subsequent needle biopsy voids $(B, C)$ are defined for image analysis. $(E)$ Time-intensity curves (corrected for T1 heterogeneity) from each ROI are fit to a general kinetic model convolution integral using an arterial input function measured from the external iliac artery. The transfer constant $\mathrm{K}^{\text {trans }}$ (corresponding to the magnitude of the enhancement curve, unit minute ${ }^{-1}$ ) and the rate constant $\mathrm{k}^{\mathrm{ep}}$ (describing the rate of clearance, unit minute ${ }^{-1}$ ) are thought to reflect differences in the perfusion and microvascular permeability underlying each ROI, respectively. Hematoxylineosin staining shows adenocarcinoma $(D)$ corresponding in this case to higher $\mathrm{K}^{\text {trans }}$ and $\mathrm{k}^{\text {ep }}$ than benign tissue $(F)$. cDNA microarray $(G, J)$ and reverse-phase protein array $(H, I$; array probed with STAT3 antibody) analysis can be performed on the biopsy cores. (From Menard C, Susil RC, Choyke P, et al. An interventional magnetic resonance imaging technique for the molecular characterization of intraprostatic dynamic contrast enhancement. Mol Imaging 2005;4(1):63-6; with permission.)

scanner is only be possible with custom-designed interventional MR imaging tables, which is the subject of ongoing work. Finally, larger studies are required to confirm the clinical value and role of MR-guided biopsy in patients with prostate cancer.

\section{Brachytherapy}

\section{Permanent implant}

For patients with localized prostate cancer at low risk for extraprostatic extension, permanentseed brachytherapy is an accepted and effective 
minimally invasive treatment strategy. Radioactive seeds are conventionally placed and left throughout the prostate gland under ultrasound guidance using a transperineal template. One important performance measure of the procedure is the proportion of the prostate gland receiving the minimum desired dose. Treatment-related toxicity is associated with radiation dose delivered to the surrounding normal organs, including the urethra, bladder wall, rectal wall, penile bulb, and neurovascular bundles.

In an effort to avoid toxicity with permanentseed brachytherapy, investigators at Harvard University translated the conventional transperineal ultrasound technique to an open MR imaging scanner architecture [45]. Even at low field strength, the peripheral zone of the prostate gland (where most cancers are known to reside) could be distinguished from the central gland, thus permitting partial prostatic irradiation whereby permanent seeds were placed in the peripheral zone only, thereby reducing the radiation dose to the urethra and bladder wall (Fig. 9). Five-year results confirmed the equivalence of this approach to radical prostatectomy in biochemical disease-free survival [46].

Ultrasound or low-field interventional MR images, however, cannot accurately identify prostatic subsites of tumor burden that may benefit from targeted radiation dose escalation. For this reason, a number of investigators have attempted to coregister previously acquired diagnostic MR images to interventional images using techniques ranging from subjective interpretation to finite element-based deformable registration [47-52]. Permanent-seed brachytherapy performed directly in a high-field diagnostic scanner would circumvent this step and potentially reduce the error introduced by coregistration. This methodology is currently being investigated in the Netherlands, where the technical feasibility of a novel singleneedle technique has been proposed [53].

\section{Temporary implant}

Patients with intermediate- or high-risk prostate cancer have a higher intraprostatic burden of disease. A number of prospective randomized studies have confirmed that such patients may benefit from escalation of radiation dose [54-56]. By virtue of the "inverse square" law, brachytherapy "radiation boosts" result in a much steeper dose gradient and, hence, can achieve better sparing of adjacent normal structures compared with external beam radiotherapy. Such a highly desirable quality can paradoxically lead to important errors; therefore, the technique demands a high level of accuracy and precision and mandates optimal image guidance.

High dose rate temporary implants offer several advantages over permanent-seed implants. Dosimetric calculations are performed immediately following the catheter placement procedure, which permits the treatment plan to be based on the actual geometry of the implant relative to the anatomy. The treatment is immediately delivered with an afterloading technique, and problems with organ motion, setup error, and postimplant edema are circumvented. A single high-intensity ${ }^{192}$ Ir source can be placed at any position for any length of time within each needle. These two variables (dwell position and dwell time) can be optimized using computer programs designed to achieve dose distribution that conforms to the target volume, while limiting dose to normal structures at risk of radiation injury.

Investigators at the University of CaliforniaSan Francisco have manually aligned previous diagnostic MR imaging/MR spectroscopic imaging datasets to "treatment planning" CT or MR images acquired after brachytherapy catheters were inserted into the prostate gland under ultrasound guidance [57]. Based on the diagnostic images, subprostatic sites suspicious for tumor burden that were specifically targeted for further dose escalation were defined. It was found that the dose could be safely escalated to these sites without overdosing the urethra or the rectum.

To circumvent the error associated with coregistration of previously acquired diagnostic images, a technique for transperineal placement of brachytherapy catheters in a 1.5-T scanner was developed [37]. This technique is identical to the biopsy technique described previously, whereby patients are placed under general anesthesia in the left lateral decubitus position on the MR imaging table. This approach permits diagnostic images to be acquired first, followed immediately by the placement of brachytherapy catheters throughout the prostate gland. After the catheters are in place, a final diagnostic-quality $\mathrm{T} 2$-weighted image set can be acquired and directly used to plan and optimize radiation delivery [58]. The authors have used this approach to demonstrate a unique ability to limit radiation dose to the neurovascular bundle-a structure critical to sexual functionwhich is immediately adjacent to the prostate gland and best visualized on MR imaging [10]. 

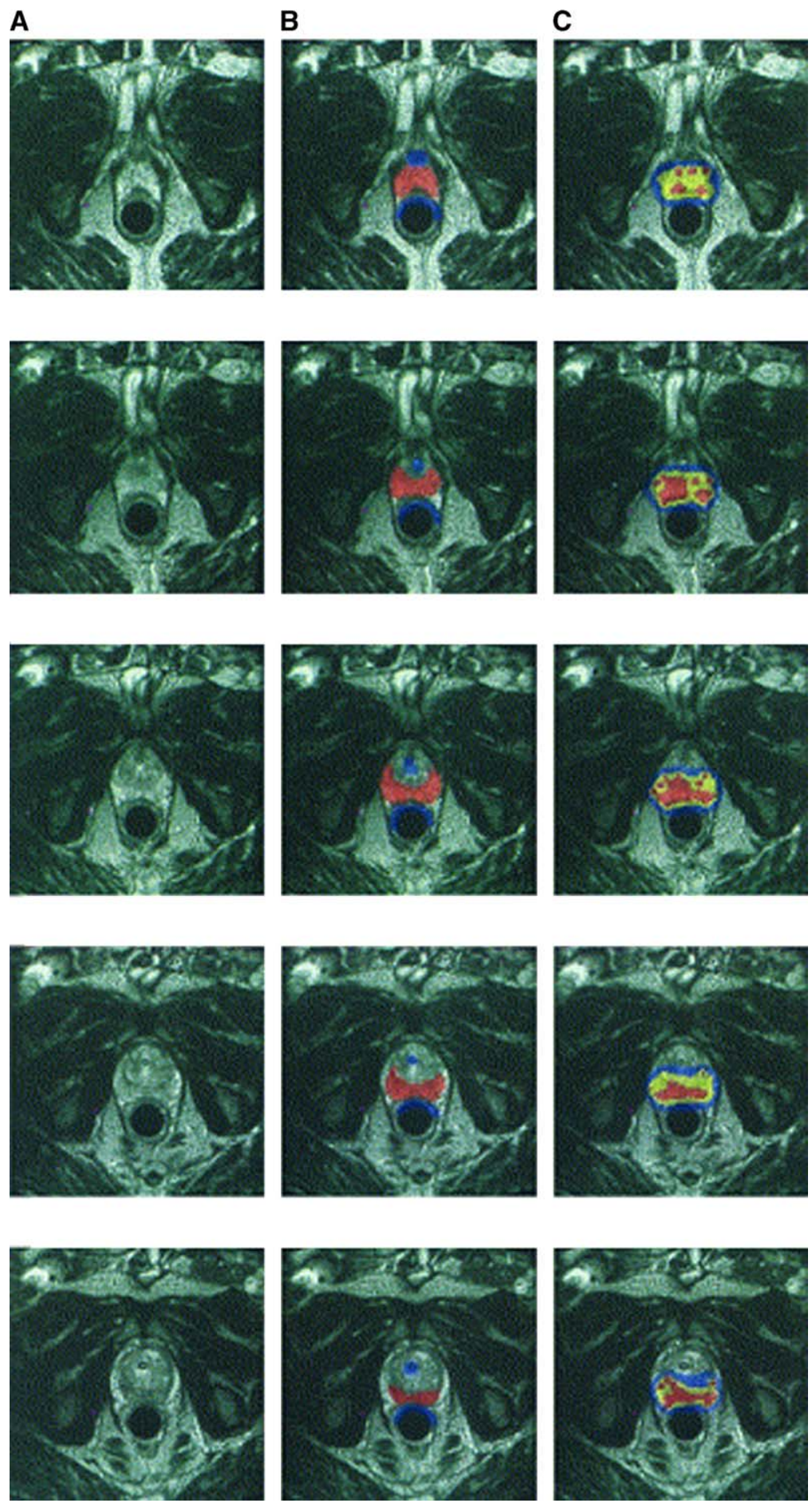

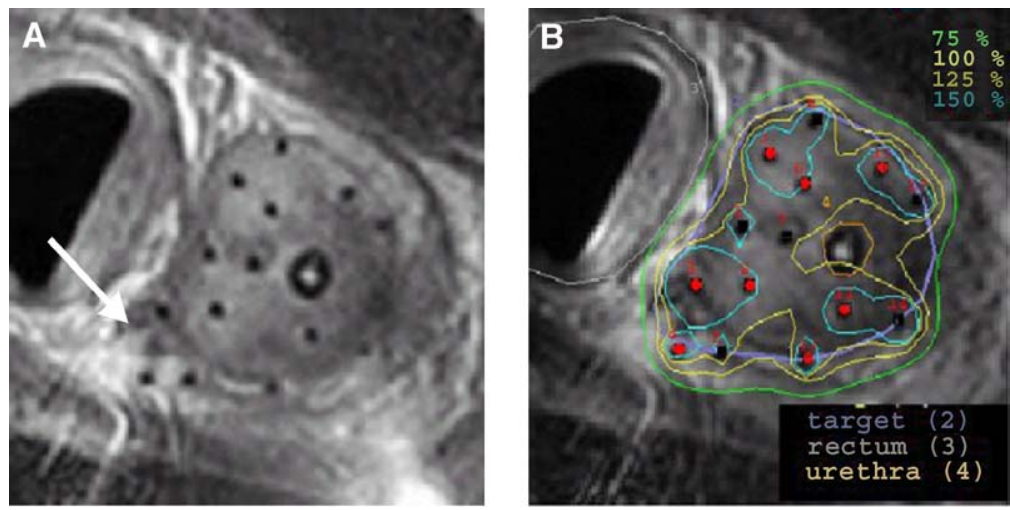

Fig. 10. High dose rate brachytherapy in a 1.5-T scanner. $(A)$ Fourteen brachytherapy catheters (signal voids) were placed throughout the prostate gland and at sites of visualized extracapsular extension (arrow). (B) Radiation was delivered according to a dosimetry plan whereby the target volume including the prostate gland and extracapsular sites of disease extension (purple line) is encompassed by $100 \%$ of the prescription dose (outer yellow outline). The urethral dose (orange outline) is kept below $125 \%$ of the prescription dose, and the rectal mucosa (white outline) is kept below $75 \%$ of the prescription dose. (From Menard C, Susil RC, Choyke P, et al. MRI-guided HDR prostate brachytherapy in standard 1.5T scanner. Int Radiat Oncol Biol Phys 2004;59(5):1420; with permission.)

This procedure may also offer a therapeutic advantage for those patients who have extracapsular extension of disease visualized on MR images, whereby extracapsular disease may be included in the radiation target volume (Fig. 10).

\section{Thermal therapy}

The role of thermal therapies for patients with prostate cancer remains investigational at this time. Beyond anatomic guidance, there is a strong rationale for integrating thermal treatment, specifically heat therapy, in the MR imaging environment where temperature can be monitored noninvasively during the procedure [59]. This treatment has been demonstrated by Chen and colleagues [60], whereby patients who had locally recurrent prostate carcinoma received percutaneous interstitial microwave thermoablation continually guided with MR imaging. Four MR imaging-compatible microwave applicators were placed in the four quadrants of the prostate gland under ultrasound guidance. Patients were transferred to MR imaging, where treatment was delivered while phase images were obtained with a rapid gradient-echo technique to derive tissue temperature change on the basis of proton-resonance frequency shift (Fig. 11).

Prostate treatments with high-intensity focused ultrasound under MR imaging guidance with a transurethral [61,62] or transrectal [63] approach have been reported in the literature only at the preclinical stage to date.

\section{Summary}

MR imaging is currently the most effective diagnostic imaging tool for visualizing the anatomy and pathology of the prostate gland. Currently, the practicality and cost effectiveness of transrectal ultrasound dominates image guidance for needlebased prostate interventions. Challenges to the integration of diagnostic and interventional MR imaging have included the lack of real-time feedback, the complexity of the imaging technique, and limited access to the perineum within the geometric constraints of the MR imaging scanner.

Fig. 9. MR-guided permanent seed brachytherapy in an open MR imaging scanner. $(A)$ Series of axial T2-weighted MR images used to identify the clinical target volume, the anterior rectal wall, and the prostatic urethra. (B) Series showing segmentation of the prostate peripheral zone, the anterior rectal wall, and the prostatic urethra. $(C)$ Series after total dose received based on final ${ }^{125}$ iodine source positions. Red, $\geq 240 \mathrm{~Gy}$; yellow, $\geq 160$ and $<240$ Gy; blue, $\geq 100$ and $<160$ Gy; no color, <100 Gy. (From D'Amico AV, Cormack R, Tempany CM, et al. Real-time magnetic resonance image-guided interstitial brachytherapy in the treatment of select patients with clinically localized prostate cancer. Int $\mathbf{J}$ Radiat Oncol Biol Phys 1998;42(3):513; with permission.) 


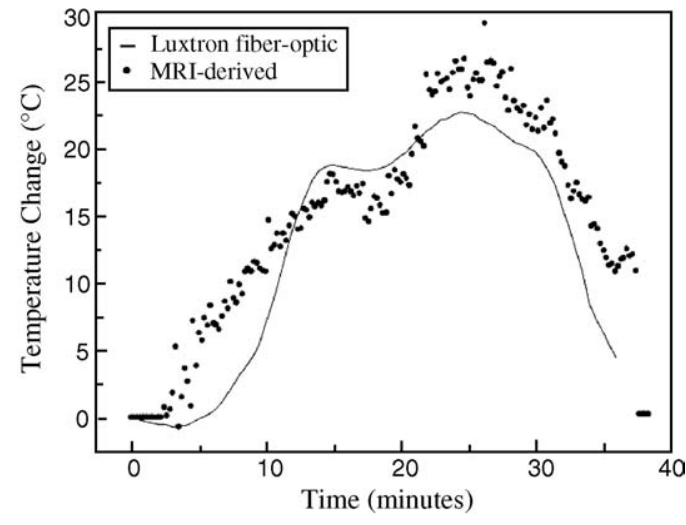

Fig. 11. Graph demonstrates the temperature measurements (Luxtron fiber-optic) and proton-resonance frequency shift temperature estimates (MR imagingderived) as a function of elapsed time (in minutes) during the prostate ablation process. Active heating began at 2 minutes and was terminated at 38 minutes. (From Chen JC, Moriarty JA, Derbyshire JA, et al. Prostate cancer: MR imaging and thermometry during microwave thermal ablation-initial experience. Radiology 2000;214(1):295; with permission.)

Two basic strategies have been explored and clinically demonstrated in the literature: (1) coregistration of previously acquired diagnostic MR imaging to interventional TRUS or open scanner MR images, and (2) stereotactic needle interventions within conventional diagnostic scanners using careful patient positioning or the aid of simple manipulators.

Currently, researchers are developing techniques that render MR imaging the method of choice for the direct guidance of many procedures. This article focuses on needle-based interventions for prostate cancer, including biopsy, brachytherapy, and thermal therapy. With rapid progress in biologic imaging of the prostate gland, the authors believe that MR imaging guidance will play an increasing role in the diagnosis and treatment of prostate cancer.

\section{References}

[1] Jemal A, Tiwari RC, Murray T, et al. Cancer statistics, 2004. CA Cancer J Clin 2004;54(1):8-29.

[2] So A, Goldenberg L, Gleave ME. Prostate specific antigen: an updated review. Can J Urol 2003;10(6): 2040-50.

[3] Bostwick DG. Gleason grading of prostatic needle biopsies. Correlation with grade in 316 matched prostatectomies. Am J Surg Pathol 1994;18(8): 796-803.
[4] Humphrey PA. Gleason grading and prognostic factors in carcinoma of the prostate. Mod Pathol 2004; 17(3):292-306.

[5] D'Amico AV, Renshaw AA, Cote K, et al. Impact of the percentage of positive prostate cores on prostate cancer-specific mortality for patients with low or favorable intermediate-risk disease. J Clin Oncol 2004;22(18):3726-32.

[6] Obek C, Louis P, Civantos F, et al. Comparison of digital rectal examination and biopsy results with the radical prostatectomy specimen. J Urol 1999; 161(2):494-8 [discussion: 498-9].

[7] Debois M, Oyen R, Maes F, et al. The contribution of magnetic resonance imaging to the threedimensional treatment planning of localized prostate cancer. Int J Radiat Oncol Biol Phys 1999; 45(4):857-65.

[8] Ito H, Kamoi K, Yokoyama K, et al. Visualization of prostate cancer using dynamic contrast-enhanced MRI: comparison with transrectal power Doppler ultrasound. Br J Radiol 2003;76(909):617-24.

[9] Mayr NA, Menard C, Molloy AR, et al. Computed tomography and magnetic resonance imaging of the prostate, uterus, and cervix. In: Chao CKS, editor. Practical essentials of intensity modulated radiation therapy. Philadelphia: Lippincott Williams \& Wilkins; 2004. p. 47-61.

[10] Citrin D, Ning H, Guion P, et al. Inverse treatment planning based on MRI for HDR prostate brachytherapy. Int J Radiat Oncol Biol Phys 2005;61(4): 1267-75.

[11] Cheng GC, Chen MH, Whittington R, et al. Clinical utility of endorectal MRI in determining PSA outcome for patients with biopsy Gleason score 7, PSA $<$ or $=10$, and clinically localized prostate cancer. Int J Radiat Oncol Biol Phys 2003;55(1): 64-70.

[12] D'Amico AV, Whittington R, Malkowicz SB, et al. Combined modality staging of prostate carcinoma and its utility in predicting pathologic stage and postoperative prostate specific antigen failure. Urology 1997;49(Suppl 3A):23-30.

[13] D'Amico AV, Schnall M, Whittington R, et al. Endorectal coil magnetic resonance imaging identifies locally advanced prostate cancer in select patients with clinically localized disease. Urology 1998;51(3):449-54.

[14] D'Amico AV, Whittington R, Malkowicz SB, et al. Combination of the preoperative PSA level, biopsy Gleason score, percentage of positive biopsies, and MRI T-stage to predict early PSA failure in men with clinically localized prostate cancer. Urology 2000;55(4):572-7.

[15] Nguyen PL, Whittington R, Koo S, et al. Quantifying the impact of seminal vesicle invasion identified using endorectal magnetic resonance imaging on PSA outcome after radiation therapy for patients with clinically localized prostate cancer. Int J Radiat Oncol Biol Phys 2004;59(2):400-5. 
[16] Poulakis V, Witzsch U, De Vries R, et al. Preoperative neural network using combined magnetic resonance imaging variables, prostate specific antigen and Gleason score to predict prostate cancer stage. J Urol 2004;172(4 Pt 1):1306-10.

[17] Kurhanewicz J, Vigneron DB, Hricak H, et al. Three-dimensional H-1 MR spectroscopic imaging of the in situ human prostate with high (0.24$0.1-\mathrm{cm}^{3}$ ) spatial resolution. Radiology 1996;198(3): 795-805.

[18] Hasumi M, Suzuki K, Taketomi A, et al. The combination of multi-voxel MR spectroscopy with MR imaging improve the diagnostic accuracy for localization of prostate cancer. Anticancer Res 2003; 23(5b):4223-7.

[19] Scheidler J, Hricak H, Vigneron DB, et al. Prostate cancer: localization with three-dimensional proton MR spectroscopic imaging-clinicopathologic study. Radiology 1999;213(2):473-80.

[20] Coakley FV, Kurhanewicz J, Lu Y, et al. Prostate cancer tumor volume: measurement with endorectal MR and MR spectroscopic imaging. Radiology 2002;223(1):91-7.

[21] Carnell D, Smith R, Taylor J, et al. Evaluation of hypoxia within human prostate carcinoma using quantified BOLD MRI and pimonidazole immunohistochemical mapping. Br J Cancer 2003;88:S12.

[22] Jiang L, Zhao DW, Constantinescu A, et al. Comparison of BOLD contrast and Gd-DTPA dynamic contrast-enhanced imaging in rat prostate tumor. Magn Reson Med 2004;51(5):953-60.

[23] Issa B. In vivo measurement of the apparent diffusion coefficient in normal and malignant prostatic tissues using echo-planar imaging. J Magn Reson Imaging 2002;16(2):196-200.

[24] Chan I, Wells W, Mulkern RV, et al. Detection of prostate cancer by integration of line-scan diffusion, T2-mapping and T2-weighted magnetic resonance imaging; a multichannel statistical classifier. Med Phys 2003;30(9):2390-8.

[25] Kantoff P, Carroll PR, D'Amico AV. Prostate cancer: principles and practice. Philadelphia: Lippincott Williams \& Wilkins; 2002.

[26] Norberg M, Egevad L, Holmberg L, et al. The sextant protocol for ultrasound-guided core biopsies of the prostate underestimates the presence of cancer. Urology 1997;50(4):562-6.

[27] Levine MA, Ittman M, Melamed J, et al. Two consecutive sets of transrectal ultrasound guided sextant biopsies of the prostate for the detection of prostate cancer. J Urol 1998;159(2):471-5 [discussion: 475-6].

[28] Shinohara K, Wheeler TM, Scardino PT. The appearance of prostate cancer on transrectal ultrasonography: correlation of imaging and pathological examinations. J Urol 1989;142(1):76-82.

[29] Rabbani F, Stroumbakis N, Kava BR, et al. Incidence and clinical significance of false-negative sextant prostate biopsies. J Urol 1998;159(4): $1247-50$.
[30] Beyersdorff D, Taupitz M, Winkelmann B, et al. Patients with a history of elevated prostate-specific antigen levels and negative transrectal US-guided quadrant or sextant biopsy results: value of MR imaging. Radiology 2002;224(3):701-6.

[31] Aihara M, Wheeler TM, Ohori M, et al. Heterogeneity of prostate cancer in radical prostatectomy specimens. Urology 1994;43(1):60-6 [discussion: 66-7].

[32] Evans SM, Hahn SM, Magarelli DP, et al. Hypoxic heterogeneity in human tumors-EF5 binding, vasculature, necrosis, and proliferation. Am J Clin Oncol Cancer Clin Trials 2001;24(5):467-72.

[33] D'Amico AV, Tempany CM, Cormack R, et al. Transperineal magnetic resonance image guided prostate biopsy. J Urol 2000;164(2):385-7.

[34] D'Amico AV, Cormack RA, Tempany CM. MRIguided diagnosis and treatment of prostate cancer. N Engl J Med 2001;344(10):776-7.

[35] Zangos S, Eichler K, Engelmann K, et al. MR-guided transgluteal biopsies with an open lowfield system in patients with clinically suspected prostate cancer: technique and preliminary results. Eur Radiol 2005;15(1):174-82.

[36] Kaplan I, Oldenburg NE, Meskell P, et al. Real time MRI-ultrasound image guided stereotactic prostate biopsy. Magn Reson Imaging 2002;20(3):295-9.

[37] Susil RC, Camphausen K, Choyke P, et al. System for prostate brachytherapy and biopsy in a standard 1.5 T MRI scanner. Magn Reson Med 2004;52(3): 683-7.

[38] Susil RC, Krieger A, Derbyshire JA, et al. System for MR image-guided prostate interventions: canine study. Radiology 2003;228(3):886-94.

[39] Beyersdorff D, Winkel A, Hamm B, et al. MR imaging-guided prostate biopsy with a closed MR unit at 1.5 T: initial results. Radiology 2005;234:576-81.

[40] Krieger A, Susil RC, Menard C, et al. Design of a novel MRI compatible manipulator for image guided prostate interventions. IEEE Trans Biomed Eng 2005;I52(2):306-13.

[41] Menard C, Susil RC, Choyke P, et al. An interventional magnetic resonance imaging technique for the molecular characterization of intraprostatic dynamic contrast enhancement. Mol Imaging 2005;4(1):63-6.

[42] Mah D, Freedman G, Milestone B, et al. Measurement of intrafractional prostate motion using magnetic resonance imaging. Int $\mathbf{J}$ Radiat Oncol Biol Phys 2002;54(2):568-75.

[43] Kitamura K, Shirato H, Seppenwoolde Y, et al. Three-dimensional intrafractional movement of prostate measured during real-time tumor-tracking radiotherapy in supine and prone treatment positions. Int J Radiat Oncol Biol Phys 2002;53(5): 1117-23.

[44] Bayley AJ, Catton CN, Haycocks T, et al. A randomized trial of supine vs. prone positioning in patients undergoing escalated dose conformal 
radiotherapy for prostate cancer. Radiother Oncol 2004;70(1):37-44.

[45] D'Amico AV, Cormack R, Tempany CM, et al. Real-time magnetic resonance image-guided interstitial brachytherapy in the treatment of select patients with clinically localized prostate cancer. Int J Radiat Oncol Biol Phys 1998;42(3):507-15.

[46] D'Amico AV, Tempany CM, Schultz D, et al. Comparing PSA outcome after radical prostatectomy or magnetic resonance imaging-guided partial prostatic irradiation in select patients with clinically localized adenocarcinoma of the prostate. Urology 2003; 62(6):1063-7.

[47] Reynier C, Troccaz J, Fourneret P, et al. MRI/ TRUS data fusion for prostate brachytherapy. Preliminary results. Med Phys 2004;31(6):1568-75.

[48] Bharatha A, Hirose M, Hata N, et al. Evaluation of three-dimensional finite element-based deformable registration of pre- and intraoperative prostate imaging. Med Phys 2001;28(12):2551-60.

[49] Mizowaki T, Cohen GN, Fung AY, et al. Towards integrating functional imaging in the treatment of prostate cancer with radiation: the registration of the MR spectroscopy imaging to ultrasound/CT images and its implementation in treatment planning. Int J Radiat Oncol Biol Phys 2002;54(5): 1558-64.

[50] Clarke DH, Banks SJ, Wiederhorn AR, et al. The role of endorectal coil MRI in patient selection and treatment planning for prostate seed implants. Int J Radiat Oncol Biol Phys 2002;52(4): 903-10.

[51] Wu X, Dibiase SJ, Gullapalli R, et al. Deformable image registration for the use of magnetic resonance spectroscopy in prostate treatment planning. Int $\mathbf{J}$ Radiat Oncol Biol Phys 2004;58(5):1577-83.

[52] DiBiase SJ, Hosseinzadeh K, Gullapalli RP, et al. Magnetic resonance spectroscopic imaging-guided brachytherapy for localized prostate cancer. Int $\mathbf{J}$ Radiat Oncol Biol Phys 2002;52(2):429-38.

[53] Van Gellekom MP, Moerland MA, Battermann JJ, et al. MRI-guided prostate brachytherapy with single needle method-a planning study. Radiother Oncol 2004;71(3):327-32.

[54] Pollack A, Zagars GK, Smith LG, et al. Preliminary results of a randomized radiotherapy dose-escala- tion study comparing $70 \mathrm{~Gy}$ with $78 \mathrm{~Gy}$ for prostate cancer. J Clin Oncol 2000;18(23):3904-11.

[55] Zeitman A, DeSilvio M, Slater JD, et al. A randomized trial comparing conventional dose (70.2GyE) and high-dose (79.2GyE) conformal radiation in early stage adenocarcinoma of the prostate: results of an interim analysis of PROG 95-09. In: Cox J, editor. ASTRO 2004. Atlanta (GA): Elsevier; 2004. p. S131-2.

[56] Sathya J, Davis I, Julian J, et al. A randomized trial comparing a conbination of iridium implant and external beam radiation to external beam radiation alone for patients with locally advanced prostate cancer. In: Cox J, editor. ASTRO 2004. Atlanta (GA): Elsevier; 2004. p. S448.

[57] Pouliot J, Kim Y, Lessard E, et al. Inverse planning for HDR prostate brachytherapy used to boost dominant intraprostatic lesions defined by magnetic resonance spectroscopy imaging. Int J Radiat Oncol Biol Phys 2004;59(4):1196-207.

[58] Menard C, Susil RC, Choyke P, et al. MRI-guided HDR prostate brachytherapy in standard $1.5 \mathrm{~T}$ scanner. Int J Radiat Oncol Biol Phys 2004;59(5): 1414-23.

[59] Peters RD, Chan E, Trachtenberg J, et al. Magnetic resonance thermometry for predicting thermal damage: an application of interstitial laser coagulation in an in vivo canine prostate model. Magn Reson Med 2000;44(6):873-83.

[60] Chen JC, Moriarty JA, Derbyshire JA, et al. Prostate cancer: MR imaging and thermometry during microwave thermal ablation-initial experience. Radiology 2000;214(1):290-7.

[61] Diederich CJ, Stafford RJ, Nau WH, et al. Transurethral ultrasound applicators with directional heating patterns for prostate thermal therapy: in vivo evaluation using magnetic resonance thermometry. Med Phys 2004;31(2):405-13.

[62] Ross AB, Diederich CJ, Nau WH, et al. Highly directional transurethral ultrasound applicators with rotational control for MRI-guided prostatic thermal therapy. Phys Med Biol 2004;49(2):189-204.

[63] Sokka SD, Hynynen KH. The feasibility of MRIguided whole prostate ablation with a linear aperiodic intracavitary ultrasound phased array. Phys Med Biol 2000;45(11):3373-83. 